\title{
Charges as Integrals over Densities: An Alternative Formulation of Coleman's Theorem
}

\author{
Eldad Gal-Ezer \\ Department of Physics and Astronomy, Tel-Aviv University, Tel-Aviv, Israel
}

Helmut Reeh

Institut für Theoretische Physik der Universität Göttingen, Göttingen, Federal Republic of Germany

Received September 10, 1974; in revised form March 9, 1975

\begin{abstract}
The charge operator is hermitean if and only if the vacuum is invariant. In that case the charge must be invariant under time translations.
\end{abstract}

Consider within the Wightman framework a quantized real field $t^{v m}(x)$ transforming under the inhomogeneous Lorentz group (ILG) according to

$$
t^{\mu m}(x) \rightarrow U(a, \Lambda) t^{\mu m} U^{-1}(a, \Lambda)=\left(\Lambda^{-1}\right)_{\mu^{\prime}}^{\mu} D_{m^{\prime}}^{m}\left(\Lambda^{-1}\right) t^{\mu^{\prime} m^{\prime}}(\Lambda x+a) .
$$

Here $m$ stands for a collection of vector indices, $U(a, \Lambda)$ is the unitary representation of the ILG, $D_{m^{\prime}}^{m}(\Lambda)$ a finite dimensional irreducible representation of the homogeneous Lorentz group (HLG) ${ }^{1}$. Let $\vartheta_{r}(\vec{x})=\vartheta\left(\frac{|\vec{x}|}{r}\right), r>0, \eta(x)$ be real test functions with compact support and $\vartheta(s)=1$ for $s \leqq 1, \int \eta\left(x^{0}\right) d x^{0}=1$ (see e.g. [4]). We assume in addition that the Fourier-transform $\tilde{\eta}\left(p^{0}\right) \neq 0$ for all finite $p^{0}$ (this is always attainable, e.g., by a small shift in imaginary direction in $p^{\circ}$-space). This rather technical looking assumption turns out to be necessary for not loosing in the limit $r \rightarrow \infty$ a contribution of $t^{\mu m}$ concentrated on a mass shell (see also ${ }^{4}$ ).

Put

$$
\begin{aligned}
Q_{r}^{m} & =\int t^{0 m}(x) \vartheta_{r}(\vec{x}) \eta\left(x^{0}\right) d^{4} x, \\
Q_{r}^{\cdot m} & =\int \partial_{0} t^{0 m}(x) \vartheta_{r}(\vec{x}) \eta\left(x^{0}\right) d^{4} x, \\
D_{r}^{m} & =\int \partial_{\mu} t^{\mu m}(x) \vartheta_{r}(\vec{x}) \eta\left(x^{0}\right) d^{4} x .
\end{aligned}
$$

Then, due to relative locality,

$$
A \rightarrow \lim _{r \rightarrow \infty} i\left[Q_{r}^{m}, A\right]=i\left[Q_{r}^{m}, A\right]_{r \geqq r_{0}(A)}, \quad A \in \mathscr{R}
$$

defines a map on the algebra $\mathscr{R}$ of all strictly local operators, and defines an operator $Q^{m}$ by

$$
Q^{m} A \Omega=\left.\left[Q_{r}^{m}, A\right]\right|_{r \geqq r_{0}(A)} \Omega
$$

with domain $\mathscr{R} \Omega$ ( $\Omega$ denotes the unique vacuum vector).

1 We consider only single-valued representations. Therefore we may assume the $t^{v m}$ to be hermitean. Mutatis mutandis the following remains unchanged for complex fields.

2 This is possible because $\Omega$ is separating. 
$Q^{m}$ should be a candidate for a "charge" operator. Therefore one would like to have $Q^{m}$ hermitean and selfadjoint.

Theorem 1. $Q^{m}$ is hermitean if and only if $\left(\Omega\left|\left[Q_{r}^{m}, A\right]\right|_{r \geqq r_{0}(A)} \Omega\right)$...0 (i.e. invariance of the vacuum).

Proof. Since $t^{\mu m}$ is assumed to be hermitean, it is straightforward to show that invariance of the vacuum implies $Q^{m}$ to be hermitean. For the other direction consider $C \in \mathscr{R}$ with $\lim _{r \rightarrow \infty}\left(\Omega \mid\left[Q_{r}^{m}, C\right] \Omega\right) \neq 0$. Assume $\Omega \in \vartheta_{Q^{m *}}$. Then

$$
\left(Q^{m^{*}} \Omega \mid C \Omega\right)=\left(\Omega \mid Q^{m} C \Omega\right)=\lim _{r \rightarrow \infty}\left(\Omega \mid\left[Q_{r}^{m}, C\right] \Omega\right) \neq 0 .
$$

On the other hand, $Q^{m} \Omega=\lim \left[Q_{r}^{m}, 1\right] \Omega=0$. Therefore $Q^{m}$ cannot be hermitean. It is easy to show slightly more, namely in case the vacuum is not invariant, $Q^{m}$ is not closable.

We see: $Q^{m}$ is a condidate for a charge precisely in case the vaccum is invariant. With certain additional assumptions one can then even show that $Q^{m}$ is selfadjoint $[5,6]$. We do not want to discuss this point here.

In case the vacuum is not invariant, one may question whether (1) is intuitively speaking a reasonable definition. To our mind, this seems to be the case, since one wants to see the action of the map transforming different fields into each other on the state space, too. Unclosable operators, however, are rather nasty objects.

Coleman [1] has shown in case of a covariant vector field, that the invariance of the vacuum implies $\partial_{\mu} j^{\mu}(x)=0$. In [3] this was somewhat generalized and shown that the conclusion does not hold in case of arbitrary tensor currents. In general one gets only $\partial_{\nu} \partial^{v} \partial_{\mu} t^{\mu m}(x)=0$. The essential aspect, however, of Coleman's theorem is that the existence of a charge operator implies that the charge operator must be independent of time. In the subsequent part of the present note it will be shown that this latter conclusion stays true for all tensor currents.

Consider $^{3}$

$$
Q^{\cdot m}=i\left[P^{0}, Q^{m}\right] \text {. }
$$

Then by using (1) one gets

$$
\begin{aligned}
Q^{\cdot m} A \Omega & =\lim _{r \rightarrow \infty}\left[Q_{r}^{\cdot m}, A\right] \Omega=\left.\left[Q_{r}^{\cdot m}, A\right]\right|_{r \geqq r_{0}(A)} \Omega \\
& =\lim _{r \rightarrow \infty}\left[D_{r}^{m}, A\right] \Omega=\left.\left[D_{r}^{m}, A\right]\right|_{r \geqq r_{0}(A)} \Omega .
\end{aligned}
$$

[For the last line one makes use of the relative locality between $t^{\mu m}$ and $A$ and of the shape of $\vartheta_{r}(\vec{x})$.] We want to show that

$$
\lim _{r \rightarrow \infty}\left(\Omega \mid\left[Q_{r}^{m}, A\right] \Omega\right)=0 \quad \forall A \in \mathscr{R}
$$

implies

$$
\left[Q_{r}^{\cdot m}, A\right]=\left[D_{r}^{m}, A\right]=0
$$

for sufficiently larger and all $A \in \mathscr{R}$.

$3 P^{0}$ stands for the Hamiltonian. We are a little sloppy in the following by not specifying precisely the domain properties of the unbounded operators involved. However, this would not change the conclusion and can easily be filled in an obious way. 
Let $B \in \mathscr{R}$ and $A_{0}$ be arbitrary, then

$$
\begin{aligned}
\left(A_{0}^{*} \Omega \mid\left\{D_{r}^{m} B-B D_{r}^{m}\right\} \Omega\right) & =\left(\Omega \mid\left\{A_{0} D_{r}^{m} B-A_{0} B D_{r}^{m}\right\} \Omega\right) \\
& =\left(\Omega \mid\left\{\left[A_{0}, D_{r}^{m}\right] B+\left[D_{r}^{m}, A_{0} B\right]\right\} \Omega\right) .
\end{aligned}
$$

We intend to show that this vanishes for a dense set of vectors $A_{0}^{*} \Omega$. Consider the second term first. For $C=A_{0} B \in \mathscr{R}$

$$
\left(\Omega \mid\left[D_{r}^{m}, C\right] \Omega\right)
$$

vanishes for large $r$ [because then

$-i\left(\Omega \mid\left[D_{r}^{m}, C\right] \Omega\right)=\left(\Omega \mid\left[\left[P_{0}, Q_{r}^{m}\right], C\right] \Omega\right)=-\left(\Omega \mid\left[\left[Q_{r}^{m}, C\right], P_{0}\right] \Omega\right)-\left(\Omega \mid\left[\left[C, P_{0}\right], Q_{r}^{m}\right] \Omega\right)$

and $P_{0} \Omega=0$, and Eq. (2)]. On the other hand, assuming Eq. (2), it was shown in [3], Proposition 2.1.1, that $\partial_{\mu} t^{\mu m}(x)$ is a free zero mass field ${ }^{4}$. Therefore only mass zero states contribute as intermediate states in (5) and one has the

Lemma 1. Let $\partial_{\mu} t^{\mu m}(x)$ transform according to an irred. rep. of the HLG. Let $C$ be local relative to $\partial_{\mu} t^{\mu m}(x)^{5}$. Then (2) implies that (5) vanishes.

Remark. Besides of the assumptions listed, we use Wightman's framework of quantum field theory. In particular the assumption of definite metric in the representation space is important.

Proof. As said above, (2) implies $D_{r}^{m} \Omega$ is a mass zero state (or vanishes). Let the irreducible rep. of the HLG be of type $(b+h, b)$.

i) Case $2 b+h \geqq 2$. Lemma 1 of [4] implies $\left\|D_{r}^{m} \Omega\right\|<\infty$. Lemma 2 of [4] then proves that (5) vanishes. (Actually, Lemma 1 of [4] was formulated for charge integrals, i.e. integrals over a zero component of a tensor. The proof, however, presented in [4] covers the present case too.)

ii) Case $(0,0), \partial_{\mu} t^{\mu m}$ is a scalar field. From 2.1.7 or 3.1.4 of [3] one gets $\partial_{\mu} t^{\mu m}(x)=0$ (the original version of Coleman's theorem), thus (4) vanishes.

iii) Case $\left(\frac{1}{2}, \frac{1}{2}\right)$. Then $t^{\mu m}$ is a second rank tensor field and 2.1 .9 of [3] gives $\partial_{\mu} t^{\mu m}(x)=0$.

iv) Case $(1,0)$ and $(0,1), \partial_{\mu} t^{\mu m}=a^{\nu \lambda}$ is an antisymmetric tensor of rank two. It has the two point function (see 5.2 of [3])

$$
\begin{aligned}
&\left(\Omega \mid a^{\mu \nu}(x) a^{\kappa \sigma}(y) \Omega\right)=\int_{p^{0} \geqq 0} d^{4} p e^{-i p(x-y)} c^{\mu \nu \kappa \sigma} \delta\left(p^{2}\right), \\
& c^{\mu \nu \kappa \sigma}= a\left(g^{\mu \kappa} g^{v \sigma}-g^{\nu \kappa} g^{\mu \sigma}\right) \\
&+b\left(p^{\mu} p^{\sigma} g^{\nu \kappa}-p^{\nu} p^{\sigma} g^{\mu \kappa}+p^{v} p^{\kappa} g^{\mu \sigma}-p^{\mu} p^{\kappa} g^{\nu \sigma}\right) \\
&+c \varepsilon^{\mu \nu \kappa \sigma}+d\left(\varepsilon^{\mu \nu \kappa \lambda} p_{\lambda} p^{\sigma}+\varepsilon^{\mu \nu \lambda \sigma} p_{\lambda} p^{\kappa}\right) .
\end{aligned}
$$

Positivity of the metric requires $a=0$ (this may also be inferred from being the derivative $\left.\partial_{\mu} t^{\mu m}\right)$. But then $\partial_{\nu} a^{\nu \lambda}(x)=0$ too. In case $v=0$, or $\lambda=0$ Proposition 2 of [4] proves the statement. To treat the remaining case $a_{i j}, i, j \in\{1,2,3\}$ consider

$$
h_{\lambda}(x)=\left(\Omega \mid\left[a_{i \lambda}(x), C\right] \Omega\right) \text {. }
$$

${ }^{4}$ Here the assumption $\tilde{\eta}\left(p^{0}\right) \neq 0$ for all finite $p^{0}$ is used. - Compare, e.g., the case $t^{\mu m}(x)=\partial^{\mu} \phi(x)$, $\phi(x)$ a free field of mass $m>0$ and $\eta$ with $\tilde{\eta}(m)=0$.

5 i.e. for $C$ given, there exists a causal region subtended by two light cones such that $\left[\partial_{\mu} t^{\mu m}(x), C\right]=0$ for $x \notin$ that region. 
This is $C^{\infty}$ in $x$ and has causal support. If $\tilde{h}_{\mu}(p)$ denotes the Fourier-transform of $h_{\mu}$, we know that $\int \tilde{h}_{\mu}(p) \tilde{\eta}\left(p^{0}\right) d p^{0}$ is $C^{\infty}$ in $\vec{p}$. On the other hand, $\partial^{\lambda} a_{i \lambda}(x)=0$ implies

$$
p^{\mu} \tilde{h}_{\mu}(p)=0 \text {. }
$$

Hence

$$
0=\frac{\partial}{\partial p^{j}}\left(p^{\mu} \tilde{h}_{\mu}(p)\right)=\partial_{j} p^{0} \tilde{h}_{0}(p)+\tilde{h}_{j}(p)+p^{i} \partial_{j} \tilde{h}_{i}(p)
$$

and

$$
\int \tilde{h}_{j}(p) \tilde{\eta}\left(p^{0}\right) \tilde{\vartheta}_{r}(\vec{p}) d^{4} p=-\int\left(\partial_{j} p^{0} \tilde{h}_{0}(p)\right) \vartheta_{r}(\vec{p}) \tilde{\eta}\left(p^{0}\right) d^{4} p-\int p^{i}\left(\partial_{j} \tilde{h}_{i}(p)\right) \tilde{\eta} \tilde{\vartheta}_{r} d^{4} p .
$$

To show that this vanishes for $r \rightarrow \infty$ consider the r.h.s. The second term vanishes because $\int \tilde{h}_{i}(p) \tilde{\eta}\left(p^{0}\right) d p^{0}$ is $C^{\infty}$ in $\vec{p}$ :

$$
\lim _{r \rightarrow \infty} \int p^{i}\left(\partial_{j} \tilde{h}_{i}(p)\right) \tilde{\eta}\left(p^{0}\right) \tilde{\vartheta}_{r}(\vec{p}) d^{4} p=\left.p^{i} \partial_{j} \int \tilde{h}_{i}(p) \eta\left(p^{0}\right) d p^{0}\right|_{\vec{p}=0}=0 .
$$

For the first term we know from [7] that

$$
\left.\int \tilde{h}_{0}(p) \tilde{\eta}\left(p^{0}\right) d p^{0}\right|_{\vec{p}=0}=\left.2 \int_{p^{0} \geqq 0} \tilde{h}_{0}(p) \tilde{\eta}\left(p^{0}\right) d p^{0}\right|_{\vec{p}=0}
$$

and this vanishes as shown above (case $v=0$ or $\lambda=0$ ). From $\square a_{v \mu}(x)=0$ we get

$$
\int_{p^{0} \geqq 0} p^{0} \tilde{h}_{0}(p) \tilde{\eta}\left(p^{0}\right) d p^{0}=|\vec{p}| \int_{p^{0} \geqq 0} \tilde{h}_{0}(p) \tilde{\eta}\left(p^{0}\right) d p^{0} .
$$

Hence

$$
\left.\left(\partial j \int_{p^{0} \geqq 0} p^{0} \tilde{h}_{0}(p) \tilde{\eta}\left(p^{0}\right) d p^{0}\right)\right|_{\vec{p}=0}=0 .
$$

In the same way one gets

$$
\left.\left(\partial^{j} \int_{p^{0} \leqq} p^{0} \tilde{h}_{0}(p) \tilde{\eta}\left(p^{0}\right) d p^{0}\right)\right|_{\vec{p}=0}=0 .
$$

Therefore the first term in (7) vanishes too for $r \rightarrow \infty$, and the lemma is proved.

In the cases ii), iii) of the preceding paragraph, $\partial_{\mu} t^{\mu m}(x)=0$ implies already (3). To show that (3) holds in the other cases too [where $\partial_{\mu} t^{\mu m}(x)=0$ need not be true as we know from 2.1.11 of [3]!], we argue that the first term in (4) vanishes for a suitable set of $A_{0}$. To do so we use an extra assumption which one may call asymptotic completeness:

$\partial_{\mu} t^{\mu m}(x)$ is a free field of zero mass (unless it vanishes) being relatively local with respect to $\mathscr{R}^{6}$. Thus there will be asymptotic zero mass one-particle states in the theory considered. There will be other asymptotic (and hence free) fields too. Our assumption (A.C.) is: There is a set of free fields, containing $\partial_{\mu} t^{\mu m}(x)$, with usual commutation relations. These fields smeared with testfunctions of compact support have an algebraic closure $\mathscr{R}_{0} . \mathscr{R}_{0} \Omega$ is assumed to be dense in $\mathscr{R} \Omega$.

Lemma 2. Let $\partial_{\mu} \mu^{\mu m}(x)$ transform according to an irreducible representation of the H.L.G. Assume (A.C.). Then (2) entails

$$
\left(\Omega \mid\left[A_{0}, D_{r}^{m}\right] B \Omega\right)=0
$$

for sufficiently large $r, A_{0} \in \mathscr{R}_{0}, B \in \mathscr{R}$ all $\mathrm{m}$.

\footnotetext{
6 One knows from quantum electrodynamics and from the Thirring model that this may happen.
} 
Proof. Since $A_{0} \in \mathscr{R}_{0}$, the commutator $\left[D_{r}^{m}, A_{0}\right]$ can be decomposed into a sum of terms

$$
\left[D_{r}^{m}, A_{1}\right] A_{2}
$$

where $A_{1}, A_{2} \in \mathscr{R}_{0}$ and $\left[D_{r}^{m}, A_{1}\right]$ is a $C$-number, i.e.

$$
\left[D_{r}^{m}, A_{1}\right]=\left(\Omega \mid\left[D_{r}^{m}, A_{1}\right] \Omega\right) .
$$

However, this vanishes according to Lemma 1, thus (8) follows.

Lemma 1 and 2 show that (4) vanishes. For large $r$ and $B \in \mathscr{R},\left[D_{r}^{m}, B\right]$ is in $\mathscr{R}$ and independent of $r$. (A.C.) then implies $\left[D_{r}^{m}, B\right] \Omega=0$, hence $\left[D_{r}^{m}, B\right]=0$. We therefore arrived at

Theorem 2. Within the Wightman framework of q.f.th. and under the assumptions of Lemma 1 and 2, the "invariance of the vacuum"

$$
\left(\Omega \mid\left[Q_{r}^{m}, A\right] \Omega\right)=0
$$

for all $A \in \mathscr{R}, r \geqq r_{0}(A)$, all $m$, implies

$$
\left[D_{r}^{m}, A\right]=\left[Q_{r}^{\cdot m}, A\right]=0
$$

for large $r$ and all $A \in \mathscr{R}$, i.e., the symmetry transformation commutes with the time evolution.

The question, of course, arises of whether the assumption (A.C.) really is necessary for the conclusion. Up to now we were not able to show directly that for an interacting Wightman field theory $\partial_{\mu} t^{\mu m} \neq 0 \square t^{\mu m}=0$ is not possible.

\section{References}

1. Coleman, S.: J. Math. Phys. 7, 787 (1966)

2. Kastler, D., Robinson, D. W., Swieca, A. J.: Commun. math. Phys. 2, 108 (1966)

3. Gal-Ezer,E., Reeh,H.: Coleman and Coleman-Okubo Theorems in Relativistic Quantum Field Theory. Fortschr. Physik 22, 481 (1974)

4. Maison, D., Reeh, H.: Commun. math. Phys. 24, 67 (1971)

5. Kraus, K., Landau, L. J.: Commun. math. Phys. 24, 243 (1972)

6. Völkel,A.H.: On Self-Adjoint Extensions of Global Charge Operators, preprint, Rio de Janeiro 1972

7. Maison, D. : Nuovo Cimento II A, 389 (1972)

H. Reeh Institut für Theoretische Physik der Universität D-3400 Göttingen Bunsenstraße 9 Federal Republic of Germany 
